\title{
Autologous CD4 and CD8 Positive Truncated CD19-expressing Antigen Presenting T-cells
}

National Cancer Institute

\section{Source}

National Cancer Institute. Autologous CD4 and CD8 Positive Truncated CD19-expressing

Antigen Presenting T-cells. NCl Thesaurus. Code C142205.

A preparation of ex vivo expanded, autologous CD4 and CD8 positive antigen presenting T-cells (T-APCs), genetically modified with a transgene encoding a truncated form of human cluster of differentiation 19 (CD19t), with potential immunostimulating activity. Upon infusion, autologous CD19t-expressing T-APCs may stimulate the proliferation and activation of preadministered therapeutic CD19-targ eted chimeric antigen receptor Tcells (CAR-T). This may both improve the persistence of the therapeutic CAR-T-cells and prevent relapse in patients with CD19 positive leukemia or lymphoma. CD19 is a B-cell specific cell surface antigen that is expressed in all B-cell lineage malignancies. 